\title{
Resposta do capim-tanzânia à aplicação de soro ácido de leite(1)
}

\author{
Emerson de Oliveira Gheri(2), Manoel Evaristo Ferreira ${ }^{(3)}$ e Mara Cristina Pessôa da Cruz ${ }^{(3)}$
}

\begin{abstract}
Resumo - O objetivo deste trabalho foi avaliar a produção de matéria seca e a quantidade de nutrientes extraída por Panicum maximum Jacq. cv. Tanzânia adubado com soro ácido de leite. Amostras de um Argissolo foram coletadas nas profundidades de 0-20, 20-40 e 40-60 $\mathrm{cm}$ e colocadas em colunas de PVC compostas por três anéis correspondentes a cada camada. As doses de soro foram definidas com base na concentração de $\mathrm{K}$, de modo a adicionar ao solo $0,75,150,225$ e $300 \mathrm{~kg} / \mathrm{ha}$ de $\mathrm{K}_{2} \mathrm{O}$ em cada uma das três aplicações: antes da semeadura, após o primeiro e após o segundo corte. $\mathrm{O}$ experimento foi realizado em casa de vegetação em delineamento inteiramente ao acaso, durante 112 dias. Após esse período, as colunas foram desmontadas e o solo foi amostrado para análise. A aplicação de soro aumentou a produção de matéria seca; a produção máxima teórica total dos três cortes foi obtida com a aplicação acumulada de $390 \mathrm{~m}^{3} / \mathrm{ha}$. As quantidades de $\mathrm{K}, \mathrm{P}$ e Ca absorvidas pela planta e o teor de $\mathrm{K}$ no solo aumentaram significativamente com as doses de soro.
\end{abstract}

Termos para indexação: forragem, nutriente, nitrogênio, potássio, resíduo orgânico.

\section{Response of tanzânia grass to acid whey application}

\begin{abstract}
The objective of this work was to evaluate the dry matter production and nutrient uptake by Panicum maximum Jacq. cv. Tanzânia fertilized with acid whey. Soil samples of an Alfisol were collected in 0-20 cm, 20-40 cm and 40-60 cm layers and were used to fill PVC columns with three rings with $20 \mathrm{~cm}$ height, obeying the collecting depth. The doses of whey were based on its $\mathrm{K}$ content and 0 , $75,150,225$ and $300 \mathrm{~kg} / \mathrm{ha} \mathrm{K}_{2} \mathrm{O}$ were added in each application: the first one applied before sowing, the second after the first cut and the third after the second cut. The experiment was carried out in a greenhouse in a completely randomized design during 112 days. After this period, the rings of the columns were separated and soil samples were taken for analysis. The dry matter production increased with whey application and the total maximum theoretical production was obtained with the cumulative application of $390 \mathrm{~m}^{3} / \mathrm{ha}$. The K, P, and Ca uptake by the grass and soil K increased with whey application.
\end{abstract}

Index terms: forage, nutrients, nitrogen, potassium, organic wastes.

\section{Introdução}

No Brasil, a pecuária depende basicamente de plantas forrageiras para alimentação dos animais, e para uma boa formação das pastagens é necessária a aplicação, principalmente, de N, P e potássio.

(1) Aceito para publicação em 19 de março de 2003.

Extraído da dissertação de mestrado apresentada pelo primeiro autor à Universidade Estadual Paulista (Unesp), Jaboticabal, SP.

(2) Rua Miguel Ignácio, 153, CEP 14090-530 Ribeirão Preto, SP. Bolsista da Fapesp. E-mail: gheri@coc.com.br

(3) Unesp, Dep. de Solos e Adubos, Via de Acesso Prof. Paulo Donato Castellane, s/no, CEP 14884-900 Jaboticabal, SP. E-mail: evaristo@fcav.unesp.br,mcpcruz@fcav.unesp.br
Por causa do preço elevado dos adubos minerais, estudos sobre fontes alternativas de nutrientes são de grande importância. Assim, na indústria de laticínios, o soro é o principal subproduto e, segundo Morris et al. (1985), contém 1,5 g/L de N, 0,9 g/L de $\mathrm{P}_{2} \mathrm{O}_{5}$ e $1,9 \mathrm{~g} / \mathrm{L}$ de $\mathrm{K}_{2} \mathrm{O}$. Essas concentrações são parecidas com as observadas por Modler (1987), que foram de 1,3, 1,1 e 1,9 g/L de N, $\mathrm{P}_{2} \mathrm{O}_{5}$ e $\mathrm{K}_{2} \mathrm{O}$, respectivamente. Como resultado de sua composição, a demanda bioquímica de oxigênio (DBO) do soro é elevada, e o seu descarte em cursos de água pode causar sérios danos ambientais. Parkin \& Marshall (1976) constataram que o uso do soro é mais barato e eficiente do que o dos fertilizantes convencionais e que, com aplicação controlada em pastagens, além 
de aumentar a produção de forragem, evita-se a poluição dos cursos de água.

Para estimar a quantidade de soro gerada na indústria de laticínios, pode-se adotar a proporção de $9 \mathrm{~kg}$ do subproduto para cada $\mathrm{kg}$ de queijo produzido (Robbins et al., 1996). No período de 1984/85, a Nova Zelândia produziu cerca de 2,5 bilhões de litros de soro (Radford et al., 1986) e em 1993, os EUA produziram 38 bilhões de litros (Robbins et al., 1996). Em 1999, a Nova Zelândia e os EUA produziriam 2,2 e 31 bilhões de litros de soro, respectivamente (Anuário Milkbizz, 1999). No Brasil, os grandes geradores de soro são as empresas Batávia S/A, Danone S/A, CCPR/Itambé, Nestlé Brasil Ltda, Parmalat Brasil e Vigor S/A, na maioria localizadas nas regiões Sul e Sudeste. A produção brasileira de soro, em 1998, foi de cerca de 3,6 bilhões de litros (Anuário Milkbizz, 1999).

Ao compararem o efeito de soro e de fertilizantes convencionais em "bluegrass", Sharrat et al. (1959) verificaram que as maiores produções foram obtidas nos tratamentos com $156.000 \mathrm{~L} /$ ha de soro e $78.000 \mathrm{~L} /$ ha de soro mais $240 \mathrm{~kg} / \mathrm{ha}$ de hidróxido de amônio, $930 \mathrm{~kg} / \mathrm{ha}$ da fórmula $12-12-12$ e $100 \mathrm{~kg} / \mathrm{ha}$ de nitrogênio.

Segundo Parkin et al. (1986), citados por Radford et al. (1986), a aplicação de 40.000 L/ha de soro em pastagens, por meio de fertirrigação, fornece quantidades de $\mathrm{N}, \mathrm{P}$ e $\mathrm{K}$ equivalentes às recomendadas na Nova Zelândia na forma de fertilizantes convencionais, e essa quantidade de soro era mais econômica do que a aplicação de $500 \mathrm{~kg}$ de superfosfato simples mais $50 \mathrm{~kg}$ de uréia, desde que a distância entre a fazenda e o laticínio fosse de até $10 \mathrm{~km}$.

Modler (1987) analisou folhas de milho cultivado em solos que receberam $640.000 \mathrm{~L} /$ ha de soro seis meses antes do plantio e observou que os teores de $\mathrm{N}, \mathrm{K}, \mathrm{Mg}$ e $\mathrm{S}$ excediam o normal e que as quantidades de N, P e K fornecidas pelo soro aplicado correspondiam a 3,8 e 6 vezes a absorvida pelo milho para uma produção de 3,2 t/ha de grãos.

Jones et al. (1993), em casa de vegetação, avaliaram o efeito equivalente a $0,250.000,500.000$ e $1.000 .000 \mathrm{~L} /$ ha de soro na produção de grãos e de matéria seca (grãos mais parte aérea) de cevada e observaram que as maiores produções, de ambos, foram obtidas com $500.000 \mathrm{~L} /$ ha de soro.
Em razão do volume de soro aplicado no solo, grandes quantidades de $\mathrm{N}$ são adicionadas, e isso pode levar à contaminação da água. Para evitar este problema, Kelling \& Peterson (1980), citados por Modler (1987), recomendaram que não se deve aplicar mais do que 625.000 a $950.000 \mathrm{~L} /$ ha/ano de soro, o que seria suficiente para suprir as necessidades de $\mathrm{N}, \mathrm{P}$ e $\mathrm{K}$ de diversas culturas.

O objetivo deste trabalho foi avaliar a produção de matéria seca e a quantidade de nutrientes extraída por Panicum maximum Jacq. cv. Tanzânia adubado com soro ácido de leite.

\section{Material e Métodos}

O experimento foi realizado em casa de vegetação e teve por finalidade avaliar a aplicação de cinco doses de soro equivalentes a $0,75,150,225$ e $300 \mathrm{~kg} / \mathrm{ha}$ de $\mathrm{K}_{2} \mathrm{O}$. $\mathrm{O}$ delineamento experimental empregado foi o inteiramente casualizado com cinco repetições, totalizando 25 unidades experimentais.

O solo utilizado foi o Podzólico Vermelho-Amarelo, textura média (Argissolo Vermelho-Amarelo, segundo Embrapa, 1999) coletado em área de pastagem degradada de braquiária, nas profundidades de 0-20, 20-40 e 40-60 cm e cujas características químicas, determinadas segundo Raij et al. (1987), encontram-se na Tabela 1.

A partir do índice de saturação por bases inicial do solo da camada arável, foram calculadas as quantidades do carbonato de cálcio p.a. $\left(\mathrm{CaCO}_{3}\right)$ e hidroxicarbonato de magnésio p.a. $\left(4 \mathrm{MgCO}_{3} \cdot \mathrm{Mg}(\mathrm{OH})_{2} \cdot 5 \mathrm{H}_{2} \mathrm{O}\right)$ a aplicar, na proporção $\mathrm{Ca}: \mathrm{Mg}$ de 4:1, para elevar a saturação por bases (V\%) a 70\%, conforme Werner et al. (1996). Porções de solo da camada de $0-20 \mathrm{~cm}$, equivalentes a $10,5 \mathrm{dm}^{3}$, receberam as quantidades correspondentes de corretivos e $140 \mathrm{mg} / \mathrm{dm}^{3}$ de P (superfosfato triplo). O solo foi umedecido a $60 \%$ da capacidade de retenção de água, com água desionizada, e deixado em incubação por 35 dias, ajustando-se periodicamente a umidade. Nesse período as amostras receberam solução de sulfato de amônio mais nitrato de amônio, de modo a adicionar $63,2 \mathrm{mg} / \mathrm{dm}^{3}$ de $\mathrm{N}$ e $20 \mathrm{mg} / \mathrm{dm}^{3}$ de enxofre.

Após a incubação, o solo foi secado, destorroado, passado em peneira de $6 \mathrm{~mm}$ de abertura de malha, homogeneizado, e cerca de $0,7 \mathrm{dm}^{3}$ foi coletado para análise química. O V\% médio atingido foi de $66 \%$ (pH de 5,3 em $\mathrm{CaCl}_{2}$ 0,01 mol/L), o teor médio de $\mathrm{P}$ resina foi de $63 \mathrm{mg} / \mathrm{dm}^{3}$, e os de Ca e Mg foram, respectivamente, 38 e $14 \mathrm{mmol}_{\mathrm{c}} / \mathrm{dm}^{3}$.

O experimento foi realizado em colunas de PVC, cada uma constituída por três anéis com $25 \mathrm{~cm}$ de diâmetro e 
altura de $22 \mathrm{~cm}$ (o da parte superior) e de $20 \mathrm{~cm}$ (os da parte intermediária e inferior), unidos por fita adesiva, e com uma tampa ("cap") acoplada ao anel inferior. As paredes internas dos anéis receberam uma camada de resina líquida, sobre a qual foi espalhada uma mistura de areia grossa e areia fina lavadas, a fim de se evitar o escoamento preferencial de água pelas paredes da coluna. Depois de montadas, as colunas foram casualizadas e preenchidas com o solo oriundo de cada profundidade. O solo da camada de 40-60 $\mathrm{cm}$ ocupou o anel inferior; o da de 20-40 cm, o intermediário, e o da camada de $0-20 \mathrm{~cm}$, o superior. Cada coluna recebeu $29,4 \mathrm{dm}^{3}$ de solo.

O solo de cada coluna foi umedecido a $60 \%$ da capacidade de retenção de água. Cinco dias após o umedecimento, foi feita a primeira aplicação de soro em quantidades equivalentes a $0,75,150,225$ e $300 \mathrm{~kg} / \mathrm{ha}$ de $\mathrm{K}_{2} \mathrm{O}$, segundo o tratamento, e água, de modo a balancear a quantidade aplicada à do tratamento que recebeu mais soro. O soro utilizado, derivado da fabricação do queijo petit suisse, foi fornecido pela Danone S/A e transportado em galões de $5 \mathrm{~L}$ em caminhão refrigerado (Tabela 2). Apresentava odor característico e foi aplicado logo após sua chegada ao laboratório.

Três dias após a aplicação do soro foi realizada a semeadura do capim-tanzânia, e quatro dias depois a maioria das plântulas havia emergido. Aos 12 dias da emergência, foi realizado o desbaste, deixando-se quatro plantas por coluna.

Até o primeiro corte, feito aos 45 dias após a emergência, as plantas receberam duas aplicações de $100 \mathrm{mg} / \mathrm{dm}^{3}$ de $\mathrm{N}$ (nitrato de amônio). $\mathrm{O}$ corte foi feito a $10 \mathrm{~cm}$ do solo.

Três dias após o primeiro corte do capim e cinco dias após o segundo, foram realizadas a segunda e a terceira aplicações de soro, respectivamente, do mesmo modo do descrito na primeira. Entre o primeiro e o segundo cortes do capim, foram feitas três aplicações de $\mathrm{N}$, totalizando $260 \mathrm{mg} / \mathrm{dm}^{3}$. Em uma das adubações foi usado sulfato de amônio, de modo a adicionar $51,4 \mathrm{mg} / \mathrm{dm}^{3}$ de enxofre. Outro fertilizante utilizado foi o nitrato de amônio. Depois de 35 dias do primeiro corte, foi realizado o segundo corte, a $10 \mathrm{~cm}$ do solo. Entre o segundo e o terceiro cortes, também foram feitas três adubações, mantendo os $260 \mathrm{mg} / \mathrm{dm}^{3}$ de $\mathrm{N}$ e diminuindo a dose de $\mathrm{S}$ para $22,9 \mathrm{mg} / \mathrm{dm}^{3}$. Depois de 35 dias do segundo corte, foi realizado o terceiro corte, a $10 \mathrm{~cm}$ do solo.

Para viabilizar a determinação do $\mathrm{N}$ nítrico nas plantas, a colheita do experimento foi feita entre $7 \mathrm{~h}$ e $8 \mathrm{~h}$, pois, de acordo com Maynard et al. (1976), entre os fatores que influenciam o acúmulo de nitrato em plantas estão a intensidade luminosa e a temperatura. As plantas foram cortadas e, rapidamente, colocadas em sacos de plástico e mantidas em geladeira a $4^{\circ} \mathrm{C}$, até serem lavadas em água corrente, em solução de detergente neutro $(1 \mathrm{~mL} / \mathrm{L})$ e em água desionizada (por três vezes). Em seguida, as plantas foram secadas em estufa com circulação forçada de ar, com temperatura em torno de $65^{\circ} \mathrm{C}$, até atingirem peso constante, e depois foram moídas. Uma parte das amostras sofreu digestão nítrico-perclórica e nos seus extratos foram determinados os teores de $\mathrm{K}, \mathrm{Ca}, \mathrm{Mg}, \mathrm{Cu}, \mathrm{Fe}, \mathrm{Mn}$ e $\mathrm{Zn}$, por espectrofotometria de absorção atômica, e o de $\mathrm{P}$, por colorimetria. Na outra parte das amostras, após digestão sulfúrica, foi determinado o $\mathrm{N}$ total, por destilação em aparelho Kjeldahl e quantificação por titulação; o N nítrico foi determinado, segundo Cataldo et al. (1975), por extração com água desionizada e quantificação por colorimetria.

Após o terceiro corte, as colunas foram desmontadas e coletadas duas amostras de solo de cada anel. Uma delas foi conservada em freezer para posterior determinação de

Tabela 1. Caracterização química inicial do solo utilizado no experimento.

\begin{tabular}{|c|c|c|c|c|c|c|c|c|c|c|c|}
\hline $\begin{array}{l}\text { Profundidade } \\
\text { (cm) }\end{array}$ & $\begin{array}{l}\text { P resina } \\
\left(\mathrm{mg} / \mathrm{dm}^{3}\right)\end{array}$ & $\begin{array}{c}\mathrm{MO} \\
\left(\mathrm{g} / \mathrm{dm}^{3}\right)\end{array}$ & $\begin{array}{c}\mathrm{pH} \\
\left(\mathrm{CaCl}_{2}\right)\end{array}$ & K & $\mathrm{Ca}$ & $\mathrm{Mg}$ & $\begin{array}{c}\mathrm{H}+\mathrm{Al} \\
\mathrm{nmol} / \mathrm{dm}^{3}\end{array}$ & Al & SB & CTC & $\begin{array}{l}\mathrm{V} \\
(\%)\end{array}$ \\
\hline $0-20$ & 8 & 36 & 4,6 & 1,5 & 20 & 10 & 41 & 2 & 32 & 73 & 44 \\
\hline $20-40$ & 3 & 19 & 4,3 & 1,1 & 8 & 4 & 39 & 6 & 13 & 52 & 25 \\
\hline $40-60$ & 2 & 14 & 4,1 & 1,9 & 5 & 3 & 47 & 9 & 10 & 57 & 17 \\
\hline
\end{tabular}

Tabela 2. Caracterização química do soro utilizado no experimento.

\begin{tabular}{|c|c|c|c|c|c|c|c|c|c|}
\hline \multirow[t]{2}{*}{ Aplicação } & \multirow[t]{2}{*}{$\mathrm{pH}$} & \multirow[t]{2}{*}{$\mathrm{C} / \mathrm{N}$} & $\mathrm{C}$ & $\mathrm{N}$ & $\mathrm{K}$ & $\mathrm{P}$ & $\mathrm{Ca}$ & $\mathrm{Mg}$ & $\mathrm{S}$ \\
\hline & & & \multicolumn{7}{|c|}{$-------------------------(\mathrm{g} / \mathrm{L})--------------------------$} \\
\hline Primeira & 4,4 & 40 & 28 & 0,7 & 1,7 & 0,5 & 0,9 & 0,1 & 0,05 \\
\hline Segunda & 4,5 & 37 & 26 & 0,7 & 1,5 & 0,7 & 1,1 & 0,1 & 0,06 \\
\hline Terceira & 4,4 & 44 & 22 & 0,5 & 1,5 & 0,5 & 0,9 & 0,1 & 0,04 \\
\hline
\end{tabular}


N nítrico, segundo Silva (1999), e a outra foi secada ao ar para determinação de K (Raij et al., 1987) e N total (Tedesco et al., 1985).

Os dados de produção de matéria seca, concentração de $\mathrm{N}$ nítrico e de quantidade absorvida de nutrientes foram submetidos à análise de variância, a 1\% de probabilidade, $\mathrm{e}$ as médias comparadas pelo teste de Tukey, a $5 \%$ de probabilidade. Foram também realizadas análises de regressão.

\section{Resultados e Discussão}

No primeiro corte ocorreu aumento linear na produção de matéria seca de folhas, colmo + bainhas e total (Figura 1). Nos demais cortes e no total produzido, ocorreram aumentos que seguiram tendência

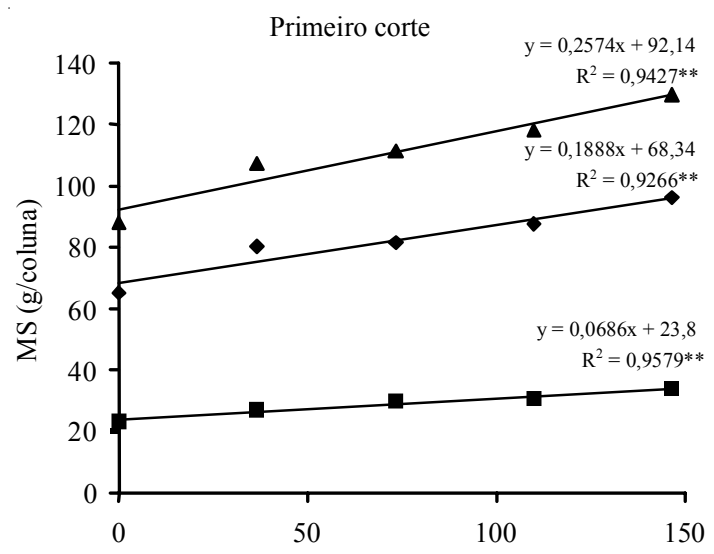

Terceiro corte

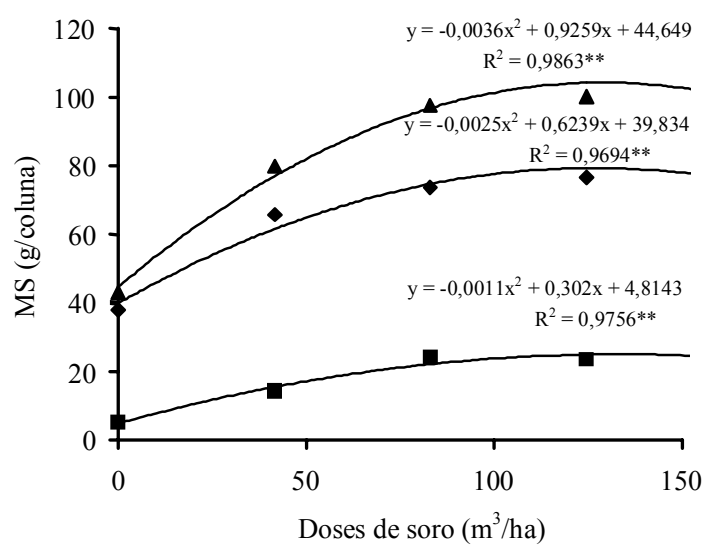

quadrática, aumentando até a dose de $110 \mathrm{~m}^{3} /$ ha de soro no primeiro corte e a reaplicação de $125 \mathrm{~m}^{3} /$ ha de soro no segundo e no terceiro cortes (que equivaleram à aplicação de $225 \mathrm{~kg} /$ ha de $\mathrm{K}_{2} \mathrm{O}$ por corte). Embora nos tratamentos que receberam 0 e $75 \mathrm{~kg} / \mathrm{ha}$ de $\mathrm{K}_{2} \mathrm{O}$ por corte tenham sido obtidas produções semelhantes de matéria seca nos dois primeiros cortes, notaram-se sintomas visuais de deficiência de $\mathrm{K}$ : clorose, da ponta para a base da folha e das bordas para o centro, seguida de necrose, inicialmente nas folhas mais velhas. No terceiro corte, a produção diminuiu em relação à dos cortes anteriores e os sintomas de deficiência de K se manifestaram nos tratamentos que receberam 0,75 e $150 \mathrm{~kg} / \mathrm{ha}$ de $\mathrm{K}_{2} \mathrm{O}$ por corte. As doses de 225 e $300 \mathrm{~kg} / \mathrm{ha}$ de

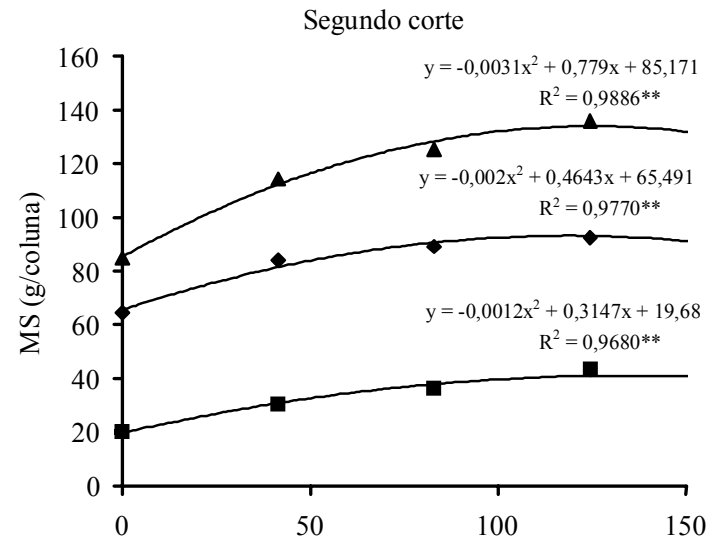

Total dos três cortes

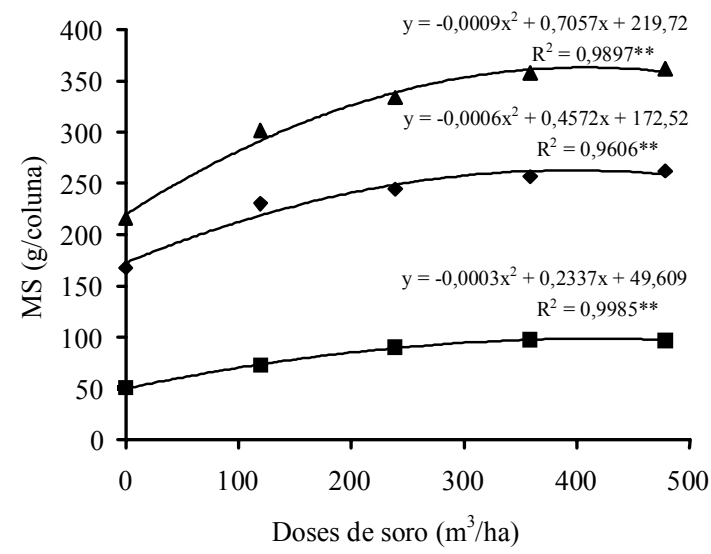

Figura 1. Efeito de doses de soro na produção de matéria seca (MS) de folhas ( $\bullet$ ), colmo + bainha $(\mathbf{\square})$ e total $(\mathbf{\Delta})$ de Panicum maximum Jacq. cv. Tanzânia, no primeiro, segundo e terceiro corte e total produzido nos três cortes. * Significativo a $1 \%$ de probabilidade pelo teste $\mathrm{F}$. 
$\mathrm{K}_{2} \mathrm{O}$ causaram diminuição de produção de matéria seca sem, contudo, aparecer sintoma de deficiência de qualquer nutriente, podendo essa diminuição ser atribuída a um desequilíbrio nutricional ou à deficiência de Mg, uma vez que N, P, K e S estavam sendo adicionados pela adubação mineral ou pelo soro. Os teores de $\mathrm{Ca}$, ao final do experimento, eram altos no solo (acima de $17 \mathrm{mmol}_{\mathrm{c}} / \mathrm{dm}^{3}$ ), e os de $\mathrm{Mg}$, muito baixos (abaixo de $2 \mathrm{mmol}_{\mathrm{c}} / \mathrm{dm}^{3}$ ).

Por meio da derivada primeira da equação de regressão, para o total produzido pelo capim-tanzânia nos três cortes, pode-se estimar que a produção máxima teórica de matéria seca ocorreu com a aplicação de $390 \mathrm{~m}^{3} /$ ha de soro, o que corresponde a $736 \mathrm{~kg} /$ ha de $\mathrm{K}_{2} \mathrm{O}$ (Figura 1). Sharrat et al. (1959) obtiveram produção máxima de "bluegrass" com a aplicação de $156 \mathrm{~m}^{3} /$ ha de soro, e Modler (1987) obteve produção de 3,2 t de grãos de milho com aplicação de $640 \mathrm{~m}^{3} /$ ha. Jones et al. (1993) conseguiram produção máxima de cevada com a aplicação de $500 \mathrm{~m}^{3} / \mathrm{ha}$ de soro.

No primeiro corte, a relação lâmina foliar:(colmo + bainha) foi de aproximadamente 3:1 em todos os tratamentos. No segundo e terceiro cortes, os tratamentos que receberam as doses menores de soro $(0 \mathrm{e}$ $75 \mathrm{~kg} /$ ha de $\mathrm{K}_{2} \mathrm{O}$ ) apresentaram essa relação maior que os demais tratamentos (Tabela 3). Resultados semelhantes foram obtidos por Forni (2000). Em relação à produção total nos três cortes a relação lâmina foliar:(colmo + bainha) foi de aproximadamente 3:1 em todos os tratamentos. Pinto et al. (1994), também em casa de vegetação, estudando os efeitos da aplicação de N, na forma de sulfato de amônio (15 e $90 \mathrm{mg} / \mathrm{kg}$ ), observaram relação menor com Panicum maximum Jacq. cv. Guiné (2:1); por sua vez, Quadros
(2001), no campo, obteve relação média de lâmina foliar:(colmo + bainha) com capim-tanzânia de aproximadamente $1: 1$, ou maior, quando menos adubado.

Os teores de $\mathrm{N}$ nítrico na planta variaram de 3.457 a $1.792 \mathrm{mg} / \mathrm{kg}$, no primeiro corte, de 1.152 a $719 \mathrm{mg} / \mathrm{kg}$, no segundo, e de 2.144 a $749 \mathrm{mg} / \mathrm{kg}$, no terceiro corte. Esses limites de valores se referem, respectivamente, ao tratamento que não recebeu soro e ao que recebeu a maior dose. Portanto, houve diminuição do teor de $\mathrm{N}$ nítrico com o aumento das doses de soro (Tabela 4), e as plantas com os maiores teores foram as que apresentaram deficiência de $\mathrm{Ke}$ menor produção de matéria seca. Nas plantas mais desenvolvidas, no tratamento que recebeu a maior aplicação de soro, a diminuição do teor de nitrato deveu-se, em parte, ao efeito de diluição, mas como a assimilação é influenciada pela nutrição da planta (Mengel \& Kirkby, 1987), aquelas com melhor suprimento em $\mathrm{K}$ devem apresentar maior eficiência na redução do íon. A ingestão de plantas com teores de nitrato entre 1.500 e $3.000 \mathrm{mg} / \mathrm{kg}$ pode provocar intoxicação em bovinos e ovinos se ela ocorrer durante três ou mais dias consecutivos (Pereira, 1992). Desse modo, os teores observados no capim-tanzânia, exceto o das plantas com deficiência aguda de K, estão abaixo do citado por Pereira (1992) e não provocariam intoxicação nesses animais.

Houve efeito linear entre a quantidade de $\mathrm{N}$ total absorvida e as doses de soro aplicadas no primeiro e segundo cortes; no primeiro, ocorreu um aumento, no segundo, uma diminuição e, no terceiro corte, houve efeito quadrático (Tabela 4). Quanto ao K, houve aumento linear no primeiro e segundo cortes e efeito quadrático no terceiro. O P apresentou efeito linear no primeiro e terceiro cortes e efeito quadrá-

Tabela 3. Relação lâmina foliar:(colmo + bainha) de capim-tanzânia em razão de doses de soro aplicadas ${ }^{(1)}$.

\begin{tabular}{|c|c|c|c|c|c|}
\hline $\begin{array}{l}\text { Soro }^{(2)} \\
\left(\mathrm{m}^{3} / \mathrm{ha}\right)\end{array}$ & $\begin{array}{c}\text { Lâmina foliar: } \\
\text { (colmo+bainha) } \\
1^{\circ} \text { corte }\end{array}$ & $\begin{array}{c}\text { Soro } \\
\left(\mathrm{m}^{3} / \mathrm{ha}\right)\end{array}$ & $\begin{array}{c}\text { Lâmina foliar: } \\
\text { (colmo+bainha) } \\
2^{\underline{0}} \text { corte }\end{array}$ & $\begin{array}{c}\text { Soro } \\
\left(\mathrm{m}^{3} / \mathrm{ha}\right)\end{array}$ & $\begin{array}{c}\text { Lâmina foliar: } \\
\text { (colmo+bainha) } \\
3^{30} \text { corte }\end{array}$ \\
\hline 0,0 & $2,8 \mathrm{a}$ & 0,0 & $3,2 \mathrm{a}$ & 0,0 & $8,7 \mathrm{a}$ \\
\hline 36,6 & $3,0 \mathrm{a}$ & 41,5 & $3,0 \mathrm{ab}$ & 41,5 & $4,8 b$ \\
\hline 73,2 & $2,8 \mathrm{a}$ & 83,0 & $2,5 \mathrm{ab}$ & 83,0 & $3,2 \mathrm{~b}$ \\
\hline 109,8 & $2,9 a$ & 124,5 & $2,2 b$ & 124,5 & $3,3 b$ \\
\hline 146,4 & $2,9 \mathrm{a}$ & 166,0 & $2,3 b$ & 166,0 & $3,3 b$ \\
\hline
\end{tabular}

${ }^{(1)}$ Em cada coluna, médias seguidas da mesma letra, na linha, não diferem entre si pelo teste de Tukey a 5\% de probabilidade; os coeficientes de variação no primeiro, no segundo e no terceiro cortes foram, respectivamente, $8,9 \%, 18,1 \%$ e $37,1 \%$. ${ }^{(2)} \mathrm{As}$ doses de $\mathrm{K}$ foram, em todas as aplicações, iguais a 0,75 , 150,225 e $300 \mathrm{~kg} / \mathrm{ha}$ de $\mathrm{K}_{2} \mathrm{O}$; houve variação na concentração de $\mathrm{K}$ no soro. 
tico no segundo. Houve aumento linear na quantidade de Ca absorvida no primeiro e terceiro cortes. Com relação ao $\mathrm{Mg}$, observou-se diminuição linear no segundo corte. O Zn e o Fe apresentaram aumento linear no primeiro e terceiro cortes, e o mesmo

Tabela 4. Relações entre teores de nitrato nas lâminas foliares (y) e quantidades de nutrientes absorvidos (y) pelo capim-tanzânia e doses do soro aplicadas (x).

\begin{tabular}{|c|c|c|}
\hline Corte & Equação & $\mathrm{R}^{2}$ \\
\hline \multicolumn{3}{|c|}{ Nitrato $(\mathrm{mg} / \mathrm{kg})$} \\
\hline $1^{\underline{0}}$ & $y=3 \cdot 175,480-10,752 x$ & $0,8790 * *$ \\
\hline $2^{\underline{o}}$ & $y=1.089,823-7,280 x+0,032 x^{2}$ & $0,7583 * *$ \\
\hline $3^{-0}$ & $y=2.003,200-25,326 x+0,111 x^{2}$ & $0,8838 * *$ \\
\hline \multicolumn{3}{|c|}{$\mathrm{N}$ total $(\mathrm{g} /$ coluna $)$} \\
\hline $1^{0}$ & $y=1,7856+0,0047 x$ & $0,8941 * *$ \\
\hline $2^{\underline{o}}$ & $y=1,7636-0,0012 x$ & $0,7082 *$ \\
\hline $3^{0}$ & $y=1,1620+0,0085 x-0,000036 x^{2}$ & $0,8874 * *$ \\
\hline \multicolumn{3}{|c|}{$\mathrm{K}(\mathrm{g} /$ coluna $)$} \\
\hline $1^{\underline{o}}$ & $y=0,6460+0,0029 x$ & $0,9358 * *$ \\
\hline $2^{\underline{o}}$ & $y=0,2572+0,0032 x$ & $0,9909 * *$ \\
\hline $3^{\mathrm{o}}$ & $\mathrm{y}=0,1387+0,0028 \mathrm{x}+0,000007 \mathrm{x}^{2}$ & $0,9991 *$ \\
\hline \multicolumn{3}{|c|}{$\mathrm{P}(\mathrm{g} /$ coluna $)$} \\
\hline $1^{\underline{0}}$ & $\mathrm{y}=0,1032+0,00049 x$ & $0,9109 * *$ \\
\hline $2^{\underline{o}}$ & $y=0,1013+0,00087 x-0,000003 x^{2}$ & $0,9233 * *$ \\
\hline $3^{\underline{0}}$ & $\mathrm{y}=0,0952+0,00056 \mathrm{x}$ & $0,9820 * *$ \\
\hline \multicolumn{3}{|c|}{$\mathrm{Ca}(\mathrm{g} /$ coluna $)$} \\
\hline $1^{\underline{0}}$ & $y=0,3112+0,00028 x$ & $0,2939 *$ \\
\hline $2^{\underline{o}}$ & & ns \\
\hline $3^{\mathrm{o}}$ & $y=0,3264+0,0012 x$ & $0,9801 * *$ \\
\hline \multicolumn{3}{|c|}{$\mathrm{Mg}(\mathrm{g} /$ coluna $)$} \\
\hline $1^{\underline{0}}$ & & \\
\hline $2^{\underline{\mathrm{o}}}$ & $y=0,3884-0,00073 x$ & $0,9419 * *$ \\
\hline $3^{\mathrm{o}}$ & & $\mathrm{ns}$ \\
\hline \multicolumn{3}{|c|}{$\mathrm{Cu}$ (mg/coluna) } \\
\hline $1^{\underline{o}}$ & & ns \\
\hline $2^{\underline{o}}$ & $y=0,4008-0,00048 x$ & $0,7771 * *$ \\
\hline $3^{\circ}$ & & ns \\
\hline \multicolumn{3}{|c|}{$\mathrm{Fe}(\mathrm{mg} /$ coluna $)$} \\
\hline $1^{\underline{0}}$ & $y=4,6596+0,0093 x$ & $0,8132 * *$ \\
\hline $2^{\underline{o}}$ & & ns \\
\hline $3^{-0}$ & $\mathrm{y}=3,4780+0,0187 \mathrm{x}$ & $0,8843 * *$ \\
\hline \multicolumn{3}{|c|}{$\mathrm{Mn}(\mathrm{mg} /$ coluna $)$} \\
\hline $1^{\underline{0}}$ & $y=7,6800+0,0159 x$ & $0,6916 * *$ \\
\hline $2^{\underline{o}}$ & $y=19,0264+0,0224 x$ & $0,6768 *$ \\
\hline $3^{\mathrm{o}}$ & $y=11,2272+0,1046 x$ & $0,9431 * *$ \\
\hline \multicolumn{3}{|c|}{$\mathrm{Zn}$ (mg/coluna) } \\
\hline $1^{\underline{o}}$ & $y=1,5868+0,0016 x$ & $0,5505 * *$ \\
\hline $2^{\underline{o}}$ & $y=1,9524-0,0015 x$ & $0,5474 *$ \\
\hline $3^{0}$ & $y=1,6928+0,0016 x$ & $0,5307 *$ \\
\hline
\end{tabular}

Pesq. agropec. bras., Brasília, v. 38, n. 6, p. 753-760, jun. 2003 efeito foi observado para Mn nos três cortes. No segundo corte, constatou-se uma diminuição linear em relação ao $\mathrm{Zn}$ e ao cobre.

O teor de nitrato do solo na testemunha foi maior do que nos tratamentos que receberam soro, provavelmente por causa da menor produção de matéria seca das plantas que ocorreu por deficiência de $\mathrm{K}$, o que ocasionou menor absorção de nitrato do solo (Tabela 5). As diferenças nos teores de nitrato entre as profundidades 0-20, 20-40 e 40-60 cm não foram significativas. Quando, na colheita do experimento, foi feita a separação dos anéis das colunas, observou-se que, apesar de estarem concentradas nos $20 \mathrm{~cm}$ superficiais, havia abundância de raízes até 40-60 $\mathrm{cm}$ de profundidade. Desse modo, mesmo que tenha havido lixiviação de nitrato, ele foi absorvido pelas raízes dos anéis inferiores.

Apesar de terem sido aplicados de 75 a $300 \mathrm{~kg} / \mathrm{ha}$ de $\mathrm{N}$ com o soro, essencialmente na forma orgânica, não houve aumento no $\mathrm{N}$ total do solo com a aplicação de soro (Tabela 5). Isso faz admitir uma taxa de mineralização dos compostos de $\mathrm{N}$ do solo suficientemente rápida a ponto de não permitir acúmulo desses compostos, e contraria a afirmação de Modler (1987), de que o $\mathrm{N}$ do soro é apenas lentamente mineralizável. $\mathrm{O}$ teor de $\mathrm{N}$ total do solo só variou com a profundidade, ou seja, na camada de $0-20 \mathrm{~cm}$ foi maior do que na camada de $20-40 \mathrm{~cm}$ e nesta maior do que na camada de 40-60 cm, acompanhando a distribuição da matéria orgânica.

O soro aumentou significativamente o teor de K no solo dos tratamentos que receberam 150, 225 e $300 \mathrm{~kg} /$ ha de $\mathrm{K}_{2} \mathrm{O}$, quando comparado aos teores no solo com doses de soro equivalentes a 0 e $75 \mathrm{~kg} / \mathrm{ha}$ de $\mathrm{K}_{2} \mathrm{O}$ (Tabela 6). Os resultados obtidos com $\mathrm{K}$ foram semelhantes aos observados por Sharrat et al. (1962), Parkin \& Marshall (1976), Jones et al. (1993) e Pivello (2001). Em relação à profundidade, o teor de $\mathrm{K}$ trocável foi maior na camada de $0-20 \mathrm{~cm}$ do que nas de 20-40 e de 40-60 cm, em todos os tratamentos. Resultados semelhantes foram encontrados por Robbins et al. (1996) em dois experimentos realizados com a adição de soro, em que ocorreu diminuição do teor de K trocável da camada de 0-30 cm para a camada de $30-60 \mathrm{~cm}$, quando se aplicou o equivalente a $0,400,800$ e $1.600 \mathrm{~m}^{3} /$ ha. 
Tabela 5. Teores médios de $\mathrm{N}$ nítrico e $\mathrm{N}$ total em amostras de solo em razão das doses de soro e de profundidades ${ }^{(1)}$.

\begin{tabular}{|c|c|c|c|c|c|c|}
\hline \multirow{2}{*}{$\begin{array}{l}\text { Profundidade } \\
(\mathrm{cm})\end{array}$} & \multicolumn{5}{|c|}{ Doses de soro $^{(2)}\left(\mathrm{m}^{3} / \mathrm{ha}\right)$} & \multirow[t]{2}{*}{ Média $^{(3)}$} \\
\hline & 0 & 119,6 & 239,2 & 358,8 & 478,4 & \\
\hline & \multicolumn{6}{|c|}{ N nítrico (mg/kg) } \\
\hline $0-20$ & 6,4 & 2,3 & 2,1 & 0,9 & 1,7 & $2,68 \mathrm{~A}$ \\
\hline $20-40$ & 9,9 & 2,2 & 0,9 & 1,0 & 1,3 & $3,06 \mathrm{~A}$ \\
\hline $40-60$ & 3,8 & 1,3 & 0,6 & 1,5 & 1,1 & $1,66 \mathrm{~A}$ \\
\hline \multirow[t]{2}{*}{ Média } & $6,70 \mathrm{~A}$ & 1,93B & $1,20 \mathrm{~B}$ & $1,13 \mathrm{~B}$ & $1,37 \mathrm{~B}$ & \\
\hline & \multicolumn{6}{|c|}{$\mathrm{N}$ total $(\mathrm{g} / \mathrm{kg})$} \\
\hline $0-20$ & 1,10 & 1,15 & 1,17 & 1,16 & 1,17 & $1,15 \mathrm{~A}$ \\
\hline $20-40$ & 0,58 & 0,54 & 0,61 & 0,56 & 0,58 & $0,57 \mathrm{~B}$ \\
\hline $40-60$ & 0,44 & 0,43 & 0,42 & 0,44 & 0,45 & $0,44 \mathrm{C}$ \\
\hline Média & $0,71 \mathrm{~A}$ & $0,71 \mathrm{~A}$ & $0,73 \mathrm{~A}$ & $0,72 \mathrm{~A}$ & $0,73 \mathrm{~A}$ & \\
\hline
\end{tabular}

(1)Médias seguidas da mesma letra, na linha ou na coluna, não diferem entre si pelo teste de Tukey a 5\% de probabilidade; os coeficientes de variação quanto aos teores de $\mathrm{N}$ nítrico e $\mathrm{N}$ total em relação às doses de soro foram de $59 \%$ e $8 \%$, respectivamente, e em relação às profundidades, de $36 \%$ e $7 \%$, respectivamente. ${ }^{(2)} \mathrm{O} \mathrm{K}$ total aplicado foi, respectivamente, $0,225,450,675$ e $900 \mathrm{~kg} / \mathrm{ha}$ de $\mathrm{K}_{2} \mathrm{O} .{ }^{(3)} \mathrm{A}$ análise estatística foi feita com dados de $\mathrm{N}$ nítrico transformados em $\sqrt{\mathrm{x}+0,5}$.

Tabela 6. Teores médios de $\mathrm{K}\left(\mathrm{mmol}_{\mathrm{c}} / \mathrm{dm}^{3}\right)$ em amostras de solo em razão de doses de soro e profundidades ${ }^{(1)}$.

\begin{tabular}{cccccc}
\hline \multirow{2}{*}{$\begin{array}{c}\text { Profundidade } \\
(\mathrm{cm})\end{array}$} & 0 & 119,6 & 239,2 & 358,8 & 478,4 \\
\cline { 2 - 5 } & $0,56 \mathrm{Ac}$ & $0,70 \mathrm{Ab}$ & $0,84 \mathrm{Aa}$ & $0,94 \mathrm{Aa}$ & $0,90 \mathrm{Aa}$ \\
$0-20$ & $0,40 \mathrm{Ba}$ & $0,42 \mathrm{Ba}$ & $0,42 \mathrm{Ba}$ & $0,44 \mathrm{Ba}$ & $0,44 \mathrm{Ba}$ \\
$20-40$ & $0,36 \mathrm{Ba}$ & $0,42 \mathrm{Ba}$ & $0,40 \mathrm{Ba}$ & $0,42 \mathrm{Ba}$ & $0,42 \mathrm{Ba}$ \\
$40-60$ & & & \multicolumn{5}{c}{ Doses de soro ${ }^{(2)}\left(\mathrm{m}^{3} / \mathrm{ha}\right)$} \\
\hline
\end{tabular}

(1)Médias seguidas pela mesma letra, maiúscula nas colunas e minúscula nas linhas, não diferem entre si pelo teste de Tukey a 5\% de probabilidade. (2) $\mathrm{O}$ K total aplicado foi, respectivamente, $0,225,450,675$ e $900 \mathrm{~kg} / \mathrm{ha}$ de $\mathrm{K}_{2} \mathrm{O}$.

\section{Conclusões}

1. A aplicação de soro ácido de leite aumenta a produção de matéria seca do capim-tanzânia; a produção máxima teórica é obtida com a aplicação de $390 \mathrm{~m}^{3} /$ ha de soro.

2. A aplicação do soro aumenta a quantidade de nutrientes absorvida pelo capim-tanzânia, particularmente de K, P e cálcio.

3. A aplicação de até $500 \mathrm{~m}^{3} /$ ha de soro ácido de leite no solo não proporciona concentrações tóxicas de nitrato nas plantas para os bovinos, nem teor de nitrato no solo em quantidade que cause contaminação do meio ambiente.

\section{Referências}

ANUÁRIO MILKBIZZ. São Paulo: Milkbizz, 1999. $326 \mathrm{p}$.

CATALDO, D. A.; HAROON, M.; SCHRAADER, L. E.; YONGS, V. L. Rapid colorimetric determination of nitrate in plant tissue by nitration of salicylic acid. Communications in Soil Science and Plant Analysis, New York, v. 6, p. 71-80, 1975.

EMBRAPA. Centro Nacional de Pesquisa de Solos (Rio de Janeiro, RJ). Sistema brasileiro de classificação de solos. Brasília: Embrapa-SPI/Embrapa-CNPS, 1999. 412 p.

FORNI, S. Disponibilidade, perfilhamento e qualidade dos cultivares Tanzânia e Mombaça de Panicum maximum Jacq. sob diferentes estratégias de adubação. 2000. 90 f. Monografia (Graduação em Zootecnia) Universidade Estadual Paulista, Jaboticabal, 2000.

JONES, S. B.; ROBBINS, C. W.; HANSEN, C. L. Sodic soil reclamation using cottage cheese (acid) whey. Arid Soil Research and Rehabilitation, London, v. 7, p. 5161, 1993.

MAYNARD, D. N.; BARKER, A. V.; MINOTTI, P. L.; PECK, N. H. Nitrate accumulation in vegetables. Advances in Agronomy, New York, v. 28, p. 71-118, 1976.

MENGEL, K.; KIRKBY, E. A. Principles of plant nutrition. $4^{\text {th }}$ ed. Bern: International Potash Institute, 1987. $687 \mathrm{p}$. 
MODLER, H. W. The use of whey as animal feed and fertilizer. Bulletin of the International Dairy Federation, Brussels, n. 212, p. 111-124, 1987.

MORRIS, S.; NIXON, J.; KILGON, R. Whey: feed or fertilizer. In: RUAKURA FARMERS' CONFERENCE, 37., 1985, Hamilton. Proceedings... Hamilton: New Zealand Ministry of Agriculture and Fisheries, 1985. p. 113-116.

PARKIN, M. F.; MARSHALL, K. R. Spray irrigation disposal of dairy factory effluent: a review of current practice in New Zealand. New Zealand Journal of Dairy Science and Technology, Palmerston North, v. 11, p. 196-205, 1976.

PEREIRA, C. A. Plantas tóxicas e intoxicações na veterinária. Goiânia: Universidade Federal de Goiás, 1992. $279 \mathrm{p}$.

PINTO, J. C.; GOMIDE, J. A.; MAESTRI, M. Produção de matéria seca e relação folha/caule de gramíneas forrageiras tropicais, cultivadas em vasos, com duas doses de nitrogênio. Revista da Sociedade Brasileira de Zootecnia, Viçosa, MG, v. 23, p. 313-326, 1994.

PIVELLO, M. A. Efeito da aplicação de soro ácido de leite nas transformações do nitrogênio e na fertilidade de solos. 2001. 44 f. Monografia (Graduação em Agronomia) - Universidade Estadual Paulista, Jaboticabal, 2001.

QUADROS, D. G. Produção e perdas de forragem em pastagens dos cultivares Tanzânia e Mombaça de Panicum maximum Jacq. adubadas com doses crescentes de NPK. 2001. 83 f. Dissertação (Mestrado em Zootecnia) - Universidade Estadual Paulista, Jaboticabal, 2001.
RADFORD, J. B.; GALPIN, D. B.; PARKIN, M. F. Utilization of whey as a fertilizer replacement for dairy pasture. New Zealand Journal of Dairy Science and Technology, Palmerston North, v. 21, p. 65-72, 1986.

RAIJ, B. van; QUAGGIO, J. A.; CANTARELLA, H.; FERREIRA, M. E.; LOPES, A. S.; BATAGLIA, O. C. Análise química do solo para fins de fertilidade. Campinas: Fundação Cargill, 1987. 170 p.

ROBBINS, C. W.; HANSEN, C. L.; ROGINSKE, M. F.; SORENSEN, D. L. Extractable potassium and soluble calcium, magnesium and potassium in two whey-treated calcareous soils. Journal of Environmental Quality, Madison, v. 25, p. 791-795, 1996.

SHARRAT, W. J.; PETERSON, A. E.; CALBERT, H. E. Effect of whey on soil and plant growth. Agronomy Journal, Madison, v. 54, p. 359-361, 1962.

SHARRAT, W. J.; PETERSON, A. E.; CALBERT, H. E. Whey as a source of plant nutrients and its effect on the soil. Journal of the Dairy Science, v. 42, p. 1126-1131, 1959.

SILVA, F. C. (Org.). Manual de análises químicas de solos, plantas e fertilizantes. Brasília: Embrapa-CNPS, 1999. $370 \mathrm{p}$.

TEDESCO, M. J.; VOLKWEISS, S. J.; BOHNEN, H. Análises de solo, plantas e outros materiais. Porto Alegre: UFRGS, 1985. p. 254-264.

WERNER, J. C.; PAULINO, V. T.; CANTARELLA, H.; ANDRADE, N. O.; QUAGGIO, J. A. Forrageiras. In: RAIJ, B. van; CANTARELLA, H.; QUAGGIO, J. A.; FURLANI, A. M. C. (Ed.). Recomendações de adubação e calagem para o Estado de São Paulo. Campinas: Instituto Agronômico, 1996. p. 263-273. (Boletim Técnico, 100) 